\title{
Variations
}

Variations

Revue internationale de théorie critique

$24 \mid 2021$

Echos

\section{Machines intelligentes et travail tertiaire}

Entretien avec Tony Smith

\section{Jason E. Smith}

Traducteur : David Buxton

\section{OpenEdition}

Journals

Édition électronique

URL : https://journals.openedition.org/variations/2028

DOI : 10.4000/variations.2028

ISSN : 1968-3960

\section{Éditeur}

Les amis de Variations

\section{Référence électronique}

Jason E. Smith, « Machines intelligentes et travail tertiaire », Variations [En ligne], 24 | 2021, mis en ligne le 03 juillet 2021, consulté le 07 juillet 2021. URL : http://journals.openedition.org/variations/ 2028 ; DOI : https://doi.org/10.4000/variations.2028

Ce document a été généré automatiquement le 7 juillet 2021.

Les ami•e•s de Variations 


\title{
Machines intelligentes et travail tertiaire
}

\author{
Entretien avec Tony Smith
}

\author{
Jason E. Smith \\ Traduction : David Buxton
}

\section{NOTE DE L'ÉDITEUR}

Initialement publié dans The Brooklyn Rail, novembre 2020.

TONY SMIтH : D'abord, félicitations pour Smart Machines and Service Work? . C'est l'un des meilleurs livres que j'ai lus sur les conséquences sociales du changement technologique. II est beaucoup plus pénétrant que les nombreux livres sur le sujet qui drainent l'attention de la grande presse. Beaucoup d'entre eux défendent un techno-utopisme, affirmant que si nous patientons un peu, et élaborons des politiques justes, les technologies avancées déclencheront une nouvelle ère de croissance et de prospérité. D'autres adoptent une position techno-dystopique, prévoyant des niveaux sans précédent de chômage et de chaos social. Comment définirais-tu ta position par rapport à ces deux pôles ?

Jason E. Smith : Les deux positions sont erronées. Elles présupposent toutes les deux que les économies capitalistes avancées subissent actuellement - ou sont sur le point de subir - une transformation technologique en profondeur, dont l'effet primaire sera un essor soudain dans la productivité du travail et de la croissance économique. Leur seule différence se trouve dans l'analyse des effets en chaîne de ce dynamisme renouvelé, les techno-dystopistes mettant l'accent sur les conséquences catastrophiques pour la stratification des classes et pour le marché du travail, entraînant une accentuation d'inégalités de revenus, et surtout un chômage de masse. Dans le livre, je me focalise sur ce dernier. Des périodes de chômage de masse résultent non du changement technologique, mais de l'effondrement économique. Si une réanimation robuste, poussée par l'automation, des économies avancées devait se réaliser, l'évidence historique suggère néanmoins une trajectoire totalement différente. On pouvait s'attendre à des bouleversements temporaires dans le marché 
du travail, alors que les procédures de production sont modifiées, les postes de travail redéfinis, la main-d'œuvre redistribuée vers des secteurs du travail intensif, des industries nouvelles créées, et une autre division du travail (à la fois sociale et technique) imposée. Aux États-Unis, il suffit de revenir sur la période s'étendant de la fin de la Seconde Guerre mondiale jusqu'à la fin des années 1960 - ce que j'appelle «Automation 1.0» - pour voir cela. Certes, sur le long terme, une telle transformation engendrera probablement un chômage croissant, et un déplacement vers le secteur des services à bas salaire, surtout dans les services à la personne. Des bouleversements et de la misère pour beaucoup, d'accord. Mais une grande transformation technologique des économies avancées n'est ni en cours, ni imminente.

Les affirmations que nos économies seraient au seuil d'une rupture technologique proviennent principalement des écoles de management, et des entreprises basées à Silicon Valley. Elles sont ensuite canalisées, remâchées, et relayées par certains journalistes et éditorialistes, accompagnées par des concepts faisant du «buzz»: "second âge des machines", "troisième révolution industrielle", "industrie 4.0 ", etc. Ce battage médiatique a fini par influencer la gauche, et par être lié à des projets de revenu de base universel, et même de "nationalisation" des réseaux sociaux, proposés dans le contexte d'une crise implacable. (En 2018, la Banque d'Angleterre a reporté que l'économie britannique avait « subi la pire décennie pour la croissance de la productivité depuis le $18^{\mathrm{e}}$ siècle $\left.»\right)$.

La rhétorique qui s'est fixée autour de l'idée d'automation fait partie d'une initiative plus large visant à alimenter une bulle boursière sans précédent, poussée principalement par une poignée d'investissements dans le secteur numérique (les FAANG, bien nommés²). L'histoire récente de l'innovation est plutôt limitée au secteur financier et à la politique monétaire: des rachats d'actions propres (800 milliards de dollars en 2018), des taux d'emprunts avoisinant l'intérêt zéro, des achats à levier massif de sociétés privées, des tours d'assouplissement quantitatif. Des tsunamis d'argent bon marché ont inondé les économies les plus riches, une grande part étant investie dans l'immobilier urbain. Avec l'arrivée de la pandémie, on a eu droit à une vague gigantesque, propulsant les bourses à des hauteurs record alors que des secteurs entiers de l'économie se sont fermés, et des dizaines de millions d'ouvriers américains ont perdu leurs emplois.

Ces fictions de changement technologique sont vitales pour une classe capitaliste qui s'imagine toujours comme une force historique progressiste, mais qui préside à une économie profondément stagnante, titubant d'une crise aiguë à la prochaine. Cette classe se présente comme une force perturbatrice, voire anarchiste, dont les innovations extraordinaires engendrent des problèmes (croissance explosive de la productivité qui rend la moitié du personnel redondante, etc.) qu'elle seule peut comprendre et résoudre (avec un revenu de base universel, les garanties à l'emploi, un New Deal vert, etc.). Il n'est pas surprenant que le buzzword de la décennie ait été "smart» (smartphones, maisons smart, usines smart, voitures smart, villes smart), terme qui reflète le narcissisme de ceux qui l'ont inventé. Pourtant, ces derniers se sont enrichis non par l'innovation, mais par la spéculation immobilière et boursière.

Que l'on ne se trompe pas, notre âge est bien celui d'un "chaos social» pour reprendre ton expression : polarisation et pulvérisation sociales, approfondissement 
de la dette et de la décroissance, des marchés de travail cassés, et conflits de classe aigus, mais fragmentés et incohérents. Mon livre essaie de prendre la mesure de ce désordre montant, et d'en offrir une explication différente.

TS: La plupart des gens croient que nous vivons dans un âge de changement technologique sans précédent. Mais dans ton livre, tu parles «d'inertie technologique soutenue ». Que veux-tu dire par cette expression frappante?

JES : Pour la plupart, les avancées technologiques qui ont émergé pendant les dernières décennies n'ont aucun impact d'une perspective macroéconomique, que ce soit de la productivité du travail, de l'emploi, des taux d'investissement, de la croissance PNB, etc. Ce n'est pas un hasard que la consolidation de cette rhétorique d'automation imminente (apprentissage machine, gouvernance algorithmique, révolution des plateformes, économie de "partage ») a coïncidé avec la montée soudaine d'entreprises comme Facebook, Apple, Alphabet (Google), Amazon, Alibaba et Tencent. Vers 2015, celles-ci ont cimenté leur statut de leaders boursiers, leurs valeurs colossales dépassant de loin les multinationales plus anciennes dans les secteurs bancaire, pétrolier, pharmaceutique et automobile, tout en s'insinuant dans la fabrique de la vie quotidienne des consommateurs. Facebook et Alphabet/Google ont promis une révolution en intelligence artificielle et en voitures sans conducteurs, alors que $90 \%$ de leurs revenus provenaient de la vente de l'espace publicitaire à d'autres entreprises (comme les banques et les fabricants d'automobiles). Ces plateformes ont accumulé des bénéfices massifs en créant et en imposant des conditions de quasi-monopole pour leurs opérations. Elles se présentent comme des entreprises de technologie, mais elles investissent relativement peu en recherche et en développement, préférant dépenser sans compter pour éliminer les concurrents potentiels, principalement en les rachetant à peine nés.

Le smartphone se propose comme l'innovation indicative de notre ère, sa marchandise «star ». Son omniprésence - dans les rues, les réunions, les cours, les salles à manger - confirme son statut emblématique. Mais il ne fait que combiner quelques appareils plus anciens comme le téléphone mobile et l'ordinateur personnel. Offrant l'accès à une panoplie de divertissements (le shopping, les plateformes de musique et de vidéo, la communication interpersonnelle) à travers un seul écran interactif, ces appareils parachèvent une confluence en cours depuis quelques décennies : la fusion du commerce et de l'information, du divertissement et de la vie sociale, de la stylisation de soi et de la vie civique, tout cela sur un écran tactile d'une taille unique. L'utilisateur est tiraillé entre ces registres, les activant tous en même temps: sans repères, sa réaction oscille entre le divertissement anecdotique et la rage inarticulée. Mais la main libre sur le marché boursier qu'ont eu les entreprises "numériques", et leur force de frappe apparente sur les loisirs, la consommation, l'identité personnelle, le discours public - tous depuis longtemps en décomposition - ont donné lieu à des affirmations concernant cette technologie de base qui dépassent de très loin son impact réel sur les habitudes de consommation, de réception, et d'interaction. Quant au travail, ces innovations promettaient de déclencher un décollage exponentiel de la productivité. C'est précisément ce qui n'est pas arrivé. Au lieu de cela, on a eu droit à des réseaux de plus en plus serrés de surveillance et de traçage, dans les rues et sur les lieux de travail.

Il est révélateur que les smartphones et les réseaux sociaux se soient décollés au milieu d'une récession profonde qui n'a jamais réussi tout à fait à "s'amortir ». 
L'iPhone fut mis sur le marché juste avant la crise financière de 2008. La façon dont les gens interagissent, s'informent, regardent des films, font du shopping ou partagent des photos devait être révolutionnée. Mais le «paradoxe de la productivité », proposé par Robert Solow en 1987, n'a jamais été démenti. Les années 2010 ont vu la croissance la plus faible de la productivité du travail depuis 1970, même dans le secteur de fabrication des biens. Le ralentissement de celle-ci a bien commencé en même temps que l'émergence du premier microprocesseur, l'Intel 4004 .

Ts : Cela m'amène à l'un des mystères les plus pérennes de l'économie contemporaine, exprimé dans la phrase «stagnation séculaire». Dans quelle mesure ton explication du décalage entre l'innovation apparente des dernières décennies et le manque relatif du dynamisme économique diffère-t-elle des autres analystes qui s'y sont penchés ?

JES : Vers la fin de 2013, au moment où la rhétorique anticipant une explosion dans la productivité poussée par l'automation commençait à chauffer, une autre fraction de la classe dirigeante étatsunienne a fait valoir une perspective très différente. Pour Larry Summers, ancien ministre des Finances sous le président Clinton, les États-Unis et d'autres économies avancées se trouvaient face à la perspective d'une stagnation profonde, où le taux de chômage élevé, la croissance ralentie du PNB, et la nonprogression des salaires persisteraient bien au-delà des ralentissements ponctuels typiques des cycles économiques.

La performance de l'économie étatsunienne semble donner raison à Summers. Le décollage promis n'a pas eu lieu. La décennie qui a vu des titres comme The Rise of the Robots ${ }^{3}$ occuper une place proéminente dans la discussion publique était également définie par une crise économique inexorable à l'échelle mondiale, la pire depuis les années 1930. Le round d'ouverture dans cette débâcle était marqué par une série d'échecs spectaculaires dans le secteur financier, alors que des banques d'investissement surendettées vacillaient, s'effondrant directement ou étant bradées à d'autres, moins exposées. Ce qui s'est déroulé ensuite était aussi prévisible que dévastateur: des années perdues avec des taux de chômage pas vus depuis des décennies, des taux d'activité plongeants, les ouvriers licenciés quittant le marché du travail, ou étant reclassés comme "infirmes». A mesure que la demande pour le travail a baissé, les salaires ont baissé aussi. Comme le travail était au repos, le capital l'était également. Pendant la décennie 2010-20, le taux d'utilisation des capacités, qui mesure la divergence entre ce qu'une économie peut théoriquement produire et sa production réelle, a atteint le niveau le plus faible depuis 1945, bien en dessous de celui de la crise des années 1970. La croissance en PNB bredouillait, alors que l'endettement du secteur privé montait en flèche. Aux États-Unis et en Europe, un phénomène d'abord observé au Japon dans les années 1990, la « décennie perdue », a refait surface: la présence fantomatique d'entreprises "zombies", survivant uniquement par le refinancement continuel de leurs dettes, alors que leur activité se réduisait. De façon encore plus significative, au moment même où tant d'éditorialistes annonçaient le nouvel âge des machines, les investissements privés ont dramatiquement chuté, atteignant un niveau plancher à un taux sans précédent depuis 1945. Les chiffres pour la productivité du travail aux États-Unis témoignaient d'un taux de croissance catastrophique, à moins de $1 \%$ annuellement, même dans le secteur industriel historiquement dynamique. 
Le rebattement des dépenses du capital a été spécialement aigu, mais ne constitue pas une aberration par rapport aux décennies précédentes. Une étude a montré que, « mesuré comme une part du PNB, l'investissement privé a décliné par trois points de pourcentage depuis 1980 ». Depuis les années 1970, seule la décennie des années 1990 se démarque comme une (petite) anomalie : une brochée d'indicateurs économiques (PNB, productivité du travail, investissements privés) a enregistré une timide remontée. Mais dans la période entre 2000 et 2011, les taux d'investissement n'ont pratiquement pas bougé, croissant à une dixième du niveau des années 1990.

Pour autant que les «stagnationnistes » jettent de la lumière sur l'assèchement des investissements privés, ils n'ont pas tort. Mais leur explication quant à pourquoi les économies développées du monde entier se sont embourbées dans une crise apparemment sans horizon, à savoir la réponse keynésienne classique d'une demande insuffisante, n'est pas convaincante. Il faut rappeler que c'est Alvin Hansen, premier disciple américain de Keynes, qui a d'abord esquissé la théorie de la stagnation séculaire en réponse au ralentissement soudain de 1937, après l'échec de la stratégie fiscale anticyclique de Roosevelt visant à soutenir la demande effondrée, et à accroître l'investissement privé. Cet échec a poussé Hansen à envisager la possibilité d'un état d'apathie chronique et intraitable, et à se demander pourquoi les économies capitalistes avancées tendaient vers l'immobilisme et la dérive (déclin de la population? fermeture des frontières?). Encore aujourd'hui, les prescriptions politiques des keynésiens sont fondées sur de nouveaux tours de dépenses déficitaires à grande échelle. Les keynésiens restent aveuglés par le succès apparent des politiques de stimulation de la demande pendant la période suivant la Seconde Guerre mondiale. Ce succès historiquement limité a permis de refouler la déroute de l'école keynésienne dans les années 1970, quand ces mêmes recettes ont contribué à créer un monstre macroéconomique - la stagflation - que les keynésiens ne pouvaient ni expliquer ni résoudre.

En 1981, la part de la dette étatique étatsunienne par rapport au PNB n'était que de $31 \%$; même avant les emprunts massifs votés en mars 2020 , ce pourcentage était plus de 100\%, proche de celui enregistré en 1945-6, quand les mécanismes de financement de la guerre étaient toujours en place. Avec l'endettement lié à la pandémie, le pourcentage est certainement plus élevé aujourd'hui. De la même manière, les dépenses étatiques comme part du PNB ont augmenté de façon constante depuis 1970, avec un pic de $43 \%$ en 2010, un an après la « sortie » de la crise de 2008. La taille de l'économie capitaliste privée continue à se rétrécir par rapport à l'activité économique globale. Ce que les économistes orthodoxes, qu'ils soient keynésiens ou néoclassiques, ont du mal à reconnaître, c'est la distinction fondamentale entre l'activité capitaliste privée et les dépenses publiques, financées par des fonds appropriés (sous la forme d'impôts ou d'emprunts) du secteur privé. Quand les gouvernements achètent des biens et des services chez les entreprises privées afin de stimuler la demande, cela pourrait aboutir à des créations d'emplois dans le court terme. Mais, comme l'a démontré avec beaucoup de clarté Paul Mattick dans Marx et Keynes $(1969)^{4}$, ce type de dépense relève simplement de la consommation à grande échelle, poussée par l'État, et financée par les bénéfices (la survaleur) générés par l'économie privée. Les dépenses étatiques de la sorte ne font que redistribuer la part des bénéfices à des capitalistes spécifiques comme Raytheon, Pfizer ou Perdue Pharma. De même, quand l'État fournit des services publics, ceux-ci ne sont pas 
vendus sur le marché, et ne produisent pas de bénéfices à réinvestir dans la production. Bien que les dépenses étatiques en éducation ou en santé s'adressent à des besoins réels, ce sont des dépenses non productives du point de vue du système capitaliste. Elles ne produisent pas de valeur (encore moins de survaleur) directement, mais sont financées par la survaleur extraite du secteur privé.

TS : Les catégories de travail «productif» et «non productif» ne se trouvent pas dans la science économique orthodoxe. Pourrais-tu parler plus de cette distinction qui joue un rôle crucial dans ton livre?

JES : Cette distinction était décisive pour l'économie politique classique, pour Smith, Ricardo et Malthus, sans oublier le grand critique de cette école, Marx. Je pense qu'elle résonne toujours dans l'expérience quotidienne des gens, comme en témoigne le succès du slogan trompeur de David Graeber des «boulots à la con " (bullshit jobs). De même, Adair Turner a récemment parlé "d'activités à somme zéro " pour caractériser la part croissante d'activités économiques consacrées non à la production de la richesse, mais à la lutte pour sa distribution ${ }^{5}$. Pourtant, cette distinction conceptuelle fondamentale échappe complètement aux économistes orthodoxes.

Ces économistes ne font pas de distinction entre activités productrices de la valeur, et activités qui la distribuent ou qui la font circuler. Ils ne voient pas non plus le besoin d'expliquer comment les profits revenant à certains types de capital - capital marchand, capital productif -représentent des parts de ce que Marx appelle la survaleur, qui a son origine dans les emplois vraiment productifs. Plutôt que de distinguer entre activités qui produisent de la valeur, et activités qui captent la survaleur redistribuée à travers la concurrence entre capitalistes, les économistes adoptent à quelques nuances près la notion courante de "productivité » utilisée par les propriétaires d'entreprises et par la presse économique. Toute activité économique qui génère des revenus est dite productive, et la productivité du travail est mesurée en divisant la production, exprimée en termes monétaires, par des unités de travail. Naturellement, l'existence d'un secteur public conséquent protégé des rigueurs de la concurrence, tout en fournissant des biens et des services non marchands, pose problème pour cette notion simpliste. On recourt à des ruses subtiles de comptabilité pour arranger les choses.

Revenons au soi-disant " paradoxe de la productivité » évoqué ci-dessus. La solution à cette énigme fut proposée, semble-t-il, par William Baumol en 1966 ( «l'effet Baumol $»)^{6}$. Celui-ci affirme qu'au fur et à mesure que certains secteurs introduisent des innovations qui permettent des économies du travail et dont l'effet net est de réduire la demande pour celui-ci, la main d'œuvre nouvellement redondante sera réallouée sans heurt à des secteurs plus intensifs en travail et moins productifs. Une grande partie de ces travailleurs seront détournés dans ce que les économistes appellent le secteur des "services ». Le modèle de Baumol prévoit qu'à mesure que les gains de productivité sont distribués inégalement à travers des secteurs technologiquement "progressistes » et des secteurs "stagnants», de plus en plus d'emplois seront concentrés dans les derniers, produisant des gains décroissants de productivité de l'ensemble de la main-d'œuvre. Extrapolée dans le long terme, cette disparité croissante des gains de productivité entre secteurs donnera une économie où la croissance de la productivité s'approche de zéro. 
Cette histoire est conceptuellement viciée. Elle dépend d'une notion de productivité qui est confuse et contradictoire, même dans ses propres termes. Dans mon livre, j'explore quelques contradictions qui surviennent quand on essaie de comparer la productivité du travail dans des secteurs différents, mesurée tantôt en unités physiques, tantôt en unités monétaires. Comment mesurer la productivité du secteur financier, difficile à caractériser en termes physiques ? Y a-t-il un sens à mesurer un secteur qui ne fait que de servir d'intermédiaire entre activités économiques, sans produire des «valeurs d'usage » consommées par des entreprises ou des ménages? Cela ne semble pas gêner les économistes. Comment mesurer la productivité d'un enseignant dans le système public, dont les prestations sont plutôt gérées localement, et ne sont pas échangées sur le marché ? Malgré les processus de travail et les fonctions sociales radicalement différents de ces exemples, ils sont réunis de façon incohérente dans la seule catégorie des « services».

D'une façon plus significative, Baumol ne discrimine pas entre activités productrices de valeur ou non. Il ne fait aucune distinction entre les biens et les services produits par le secteur public, et ceux produits par l'économie capitaliste privée, et au sein de cette dernière, entre activités productrices de valeur et activités qui ne font que distribuer ou circuler celle-ci. L'exploration de ces distinctions conceptuelles est une préoccupation centrale de mon livre. Si on utilise ces catégories, on arrive à une notion de productivité très différente de celle employée par des propriétaires et des économistes. Beaucoup d'emplois génèrent des revenus, mais ne font pas augmenter la richesse totale de la société; beaucoup d'activités qui créent des "valeurs d'usage » - fournies par l'État ou par des ménages - ne produisent pas de la valeur ou de la valeur d'échange. Un nombre significatif d'emplois dans le soi-disant secteur des services produisent de la valeur, en dépit du travail intensif et de la résistance au changement technologique; d'autres ne produisent pas de la valeur, mais peuvent faire des économies de main d'œuvre. La distinction entre travail productif et non productif traverse cette catégorie, et la rend non pertinente d'un point de vue analytique.

Cette distinction est néanmoins essentielle. Les activités non productives doivent être financées par la survaleur produite par l'économie privée; ce sont des coûts imposés par le processus d'accumulation. Les conventions de comptabilité nationale traitent ces coûts comme des revenus. L'une des tendances dans le long terme d'une économie capitaliste avancée est l'augmentation relative des activités non productives, nécessaires pour le processus d'accumulation du capital : réaliser une part du processus d'échange, faciliter l'activité par des opérations financières, et louer de la terre et de l'immobilier pour des entreprises productives. Ce surplomb croissant d'emplois qui distribuent ou font circuler de la valeur plutôt que de la créer est en même temps une condition de l'accumulation capitaliste et, à mesure que la part d'activités non productives s'accroît, une entrave à celle-ci. C'est une question épineuse, et ma pensée ici doit beaucoup à Mattick et à l'économiste Fred Moseley ${ }^{7}$ : il existe un taux de croissance de la productivité différenciable entre les dimensions productives et non productives de l'économie. Les gains de productivité dans les activités productrices de valeur (avec quelques exceptions importantes) tendent à s'accroître plus vite que celles qui distribuent de la valeur. L'expansion relative du secteur non productif qui en résulte exerce une pression écrasante vers le bas sur le taux de profit global. Le seul espoir pour soulager cette pression est une montée de la 
productivité dans le "secteur" non productif (le terme est trompeur, car la distinction entre activités productives et non productives traverse les secteurs et même les entreprises individuelles). Mais pour les raisons déjà évoquées, un tel scénario est hautement improbable, simplement parce que la baisse du taux de profit fait diminuer le taux d'investissement.

Même chez les entreprises qui en tirent directement de la survaleur du processus du travail, il n'existe pas de correspondance entre la quantité de survaleur extraite et la survaleur qui leur revient sous forme de profits. Ceux-ci reflètent la part maximale de la survaleur totale produite par l'économie globale que les entreprises arrivent à approprier dans le processus de distribution. À mesure que l'accumulation du capital se ralentit, et que les entreprises intensifient la concurrence afin d'approprier une masse déclinante de survaleur, celles-ci consacrent de plus en plus de ressources à «des activités distributives à somme zéro » (Adair Turner). Souvent, ces activités relèvent de l'encadrement, alors que la discipline accrue sur le lieu de travail exige du personnel supplémentaire pour imposer l'accélération physique de la production en l'absence d'amélioration des techniques. Mais tout aussi souvent, ces activités prennent la forme de soi-disant "services aux entreprises » : comptabilité, publicité, opérations financières, marketing. La conséquence de la guerre globale dans la distribution est de ralentir encore plus l'accumulation du capital, justement parce que ces activités représentent des coûts de fonctionnement additionnels à financer avec la somme totale de survaleur. À mesure que le taux de profit baisse, la masse déclinante de survaleur pousse les entreprises à allouer de plus en plus de ressources à l'appropriation, et non à la production de cette survaleur, ce qui fait décroître encore plus le taux de profit. C'est le dynamisme tourbillonnant d'une économie impitoyablement stagnante.

TS: À la conclusion de ton livre, tu apparais assez pessimiste concernant les syndicats et les formes de lutte au niveau local ; tu appelles à de nouvelles formes d'organisation. Sur quoi se fonde ce pessimisme? As-tu des idées sur ce à quoi pourraient ressembler ces nouvelles formes?

JES : Depuis le début du siècle, presque tous les nouveaux emplois aux États-Unis ont été créés dans les «services » à faible productivité. Des projections récentes du Bureau des Statistiques du Travail prédisent que la partie du marché du travail qui connaîtra la plus grosse expansion dans la prochaine décennie sera dans les emplois à faible salaire n'exigeant aucune qualification. C'est un scénario sinistre, qui exacerbe une dynamique établie depuis des décennies. D'une certaine manière, nous sommes toujours pris dans le courant créé par la grande vague d'innovation qui se déroula entre 1920 et 1965. J'appelle celle-ci « Automation 1.0 », mais elle comprend aussi le développement du moteur à combustion interne, l'établissement d'une infrastructure à une échelle proprement capitaliste, et les "promesses " et "périls » de l'énergie nucléaire, en plus de l'automation dans les usines. Ce n'est pas un secret que les salaires réels des ouvriers américains ont à peine bougé depuis le milieu des années 1970. Beaucoup attribuent cette stagnation des salaires à la défaite du travail organisé à partir des années 1980. Certes, le taux de syndicalisation a diminué de moitié entre-temps. Mais cette défaite n'a pas été purement politique. La situation matérielle qui a rendu possible le renforcement du pouvoir syndical dans la période après 1945 a commencé à s'éroder dès le mitan des années 1960, alors que la composition de la classe ouvrière et la nature du travail lui-même se sont mutées. La stagnation des salaires est étroitement liée au début du déclin dramatique de la 
croissance du taux de productivité. Le Bureau des Statistiques du Travail a montré que, pour la période 1973-90, la productivité des ouvriers américains s'est accrue de seulement 1,3\% annuellement, une fraction des gains enregistrés dans les deux décennies après 1945. La croissance des salaires réels exige une augmentation de la production pour chaque heure de travail. C'est pourquoi les conventions entre capital et travail aux États-Unis et en Europe ont explicitement lié les augmentations de salaire à des gains de productivité; les ouvriers et les propriétaires devaient "partager " les bénéfices d'une productivité horaire accrue. Quand de tels gains s'avèrent difficiles à réaliser, toute augmentation potentielle des salaires entraînera une baisse correspondante des bénéfices. La classe capitaliste a combattu, et combattra cette possibilité, bec et ongle.

La nature changeante du marché du travail, de la composition des classes, et du travail lui-même a eu des effets paralysants sur le mouvement ouvrier. Alors que de plus en plus de travailleurs se dirigent vers le processus de distribution, ou se concentrent dans les emplois à bas salaire du soi-disant secteur des services boutiques, centres d'appel, hôpitaux, crèches - ils se dispersent dans de multiples industries. À la différence de leurs parents et de leurs grands-parents, souvent rassemblés dans des lieux de travail à grande échelle avec des milliers de salariés, ils tendent à se disséminer dans l'espace, sur de petits sites mobilisant peu de capital fixe. S'il y a une caractéristique du secteur des services au sens large, elle est négative : le seul point en commun des processus de travail extrêmement divergents est l'intensité du travail. Une condition matérielle décisive pour la croissance aprèsguerre des syndicats, en taille et en puissance, était les effets homogénéisants de la rationalisation capitaliste de la production. Dans les périodes antérieures d'industrialisation rapide, les percées technologiques dans une industrie se généralisaient à travers les lignes de production, faisant converger les processus de travail. Des ouvriers précédemment divisés par classe, région, sexe et rémunération se retrouvaient dans des activités similaires, réduisant les différences de niveaux de compétence et de salaire. À mesure que les anciennes différenciations artisanales de compétence s'érodaient, s'externalisant dans la grande machinerie, et que la convergence des processus de travail produisait des gains importants de productivité, les travailleurs s'identifiaient plus facilement comme des travailleurs tout court, définis par rapport à la classe capitaliste, plutôt que comme des salariés d'une entreprise particulière, leurs griefs visant un patron particulier.

À mesure que les ouvriers sont expulsés des industries intensives en capital, les conditions matérielles cruciales pour la cohérence de classe disparaissent. En dépit de la spéculation des croisés de l'automation, la plupart des emplois dans le secteur des services restent imperméables - de par leur nature même - à la mécanisation. Et même quand ces emplois y sont susceptibles, les bas salaires prévalents dissuadent les propriétaires d'entreprendre des remaniements de fond en comble de ce genre d'activité (livraison, encaissement en grandes surfaces, gardiennage, sécurité, nettoyage, transport de personnes, etc.). La nature du travail lui-même (qui pour beaucoup prend la forme de services à la personne) et surtout l'absence de solidarité sont démoralisantes. Ces travailleurs n'ont pas conscience qu'ils constituent une classe dans un sens positif, qu'ils représentent quelque préfiguration d'une société à venir, à être construite dans leur image. Dans de telles conditions, un sens élevé de conflit peut se prévaloir entre eux, nourri par les facteurs d'identité (race, ethnicité, 
sexe) qui les divisent depuis longtemps. Pendant la pandémie, ces divisions incluent désormais la distinction entre ceux jugés "essentiels ", obligés de risquer leur vie pour assurer la continuité du service, ceux qui ont perdu leurs emplois, et ceux, souvent de la classe moyenne, qui ont pu migrer vers le télétravail.

Malgré l'effondrement des conditions qui ont donné lieu à l'ancien mouvement ouvrier, on a vu ces dernières années des initiatives extraordinaires de la part des travailleurs, sur les lieux de travail et dans les rues. N'oublions pas que c'est la menace d'une grève illégale par les employés de la Transportation Security Agency en 2019 qui a mis fin au non-versement des salaires par l'État fédéral. Depuis quelques années, les enseignants du système public américain ont été prêts à recourir à l'action directe, souvent dans les États conservateurs, avec du soutien populaire écrasant. Les enseignants sont restés largement protégés de la mécanisation qui a transformé d'autres industries, et leur place dans la division sociale du travail leur donne beaucoup d'influence. En France, on a eu récemment un aperçu de ce que pourrait ressembler une révolte dans « l'arrière-pays ", quand les gilets jaunes - avec toutes leurs contradictions - ont visé les centres-villes et les ronds-points. Que Dieu aide la classe capitaliste si les ouvriers dans les centres de distribution et de logistique décident d'attaquer la circulation des biens dans les ports et les artères des réseaux à flux tendus. En 2020, les troupes de la National Guard ont patrouillé les rues sujettes au couvre-feu, alors que des manifestations et des émeutes contre la police se répandaient à travers le pays, au milieu d'une pandémie létale.

Pour conclure sur une note personnelle, le pessimisme véritable, c'était de voir des centaines de milliers de gens manifester contre l'attaque à venir contre l'Iraq en 2002 et en 2003, tout en sachant notre impuissance. Malgré la misère ambiante, et malgré les traumatismes infligés par les années de crise, on est peut-être au seuil d'une réelle rupture. Quelles que soient les formes de lutte qui surgiront dans les prochaines années, il est peu probable qu'elles reviennent au modèle du mouvement ouvrier à son apogée. Face à tout ce qui s'organise contre eux, à la fois matériellement et politiquement, les travailleurs devraient avancer à tâtons vers quelque chose de nouveau.

\section{NOTES}

1. ReAktion Books, London, 2020.

2. FAANG = Facebook, Amazon, Apple, Netflix, Google. En anglais, fang signifie croc (toutes les notes sont du traducteur).

3. Martin Ford, The Rise of the Robots, Oneworld Publications, London, 2016.

4. Paul Mattick, Marx et Keynes. Les limites de l'économie mixte, (trad. Serge Bricianer), Gallimard, collection « Tel», 1972, 2010 (1969).

5. Adair Turner, Reprendre le contrôle de la dette. Pour une réforme radicale du système financier, Les Éditions de l'Atelier, 2017 (2015). 
6. William J. Baumol, William G. Bowen, Performing Arts. The Economic Dilemma : a study of problems common to theater, opera, music and dance, M.I.T. Press, Cambridge, Mass., 1966.

7. Fred Moseley, The Falling Rate of Profit in the Postwar United States Economy, St. Martins's Press, New York, 1992.

\section{AUTEURS}

JASON E. SMITH

Art Center College of Design, Los Angeles (Californie, États-Unis) 